1 Hacettepe Journal of Mathematics and Statistics

h Volume 47 (4) (2018), 813-820

\title{
$q-$ Harmonic mappings for which analytic part is $q-$ convex functions of complex order
}

\author{
Asena Çetinkaya*† and Yaşar Polatog̃lu ${ }^{\ddagger}$
}

\begin{abstract}
We introduce a new class of harmonic function $f$, that is subclass of planar harmonic mapping associated with $q$ - difference operator. Let $h$ and $g$ are analytic functions in the open unit disc $\mathbb{D}=\{z:|z|<1\}$. If $f=h+\bar{g}$ is the solution of the non-linear partial differential equation $w_{q}(z)=\frac{D_{q} g(z)}{D_{q} h(z)}=\frac{\bar{f}_{\bar{z}}}{f_{z}}$ with $\left|w_{q}(z)\right|<1, w_{q}(z) \prec b_{1} \frac{1+z}{1-q z}$ and $h$ is $q-$ convex function of complex order, then the class of such functions are called $q-$ harmonic functions for which analytic part is $q-$ convex functions of complex order denoted by $\mathcal{S}_{\mathcal{H}} e_{q}(b)$. Obviously that the class $\mathcal{S}_{\mathcal{H}_{q}(b)}$ is the subclass of $\mathcal{S}_{\mathcal{H}}$. In this paper, we investigate properties of the class $\mathcal{S}_{\mathcal{H C}_{q}(b)}$ by using subordination techniques.
\end{abstract}

Keywords: $q-$ difference operator, $q-$ harmonic mapping, $q-$ convex function of complex order.

Mathematics Subject Classification (2010): 30C45

Received : 21.03.2017 Accepted : 31.05.2017 Doi : 10.15672/HJMS.2017.480

\footnotetext{
*Department of Mathematics and Computer Sciences,İstanbul Kültür University, İstanbul, TURKEY

Email : asnfigen@hotmail.com

†Corresponding Author.

‡Department of Mathematics and Computer Sciences,İstanbul Kültür University, İstanbul, TURKEY,

Email : y.polatoglu@iku.edu.tr
} 


\section{Introduction}

A planar harmonic mapping in the open unit disc $\mathbb{D}$ is a complex valued harmonic function $f$, which maps $\mathbb{D}$ onto the some planar domain $f(\mathbb{D})$. Since $\mathbb{D}$ is a simply connected domain, the mapping $f$ has a canonical decomposition $f=h+\bar{g}$, where $h$ and $g$ are analytic in $\mathbb{D}$ and have the following power series expansions

$$
h(z)=z+\sum_{n=2}^{\infty} a_{n} z^{n} \quad \text { and } \quad g(z)=\sum_{n=1}^{\infty} b_{n} z^{n}
$$

where $a_{n}, b_{n} \in \mathbb{C}, n=0,1,2,3, \cdots$. As usual, we call $h$ the analytic part of $f$ and $g$ the co-analytic part of $f$, respectively. An elegant and complete treatment theory of the harmonic mapping is given in Duren's monograph [3]. Lewy [11] proved in 1936 that the harmonic mapping $f$ is locally univalent in $\mathbb{D}$ if and only if its Jacobian $J_{f}=$ $\left|h^{\prime}(z)\right|^{2}-\left|g^{\prime}(z)\right|^{2}$ is different from zero in $\mathbb{D}$. In view of this result, locally univalent harmonic mappings in the open unit disc are either sense-preserving if $\left|g^{\prime}(z)\right|<\left|h^{\prime}(z)\right|$ or sense-reversing if $\left|g^{\prime}(z)\right|>\left|h^{\prime}(z)\right|$ in $\mathbb{D}$. Throughout this paper, we will restrict ourselves to the study of sense-preserving harmonic mappings. We also note that $f=h+\bar{g}$ is sense-preserving in $\mathbb{D}$ if and only if $h^{\prime}$ does not vanish in $\mathbb{D}$ and the second dilatation $w(z)=\frac{g^{\prime}(z)}{h^{\prime}(z)}$ has the property $|w(z)|<1$ for all $z \in \mathbb{D}$. Therefore the class of all sensepreserving harmonic mappings in $\mathbb{D}$ with $a_{0}=b_{0}=0$ and $a_{1}=1$ will be denoted by $\mathcal{S}_{\mathcal{H}}$. Thus $\mathcal{S}_{\mathcal{H}}$ contains standard class $\mathcal{S}$ of analytic univalent functions. The family of all mappings $f \in \mathcal{S}_{\mathcal{H}}$ with the additional property that $g^{\prime}(0)=0$, i.e., $b_{1}=0$ are denoted by $\mathcal{S}_{\mathcal{H}}^{0}$. Hence it is clear that $\mathcal{S} \subset \mathcal{S}_{\mathcal{H}}^{0} \subset \mathcal{S}_{\mathcal{H}}$.

In 1908 and 1910 Jackson [8,9] initiated a study of $q$-difference operator by

$$
D_{q} f(z)=\frac{f(z)-f(q z)}{(1-q) z} \text { for } \quad z \in B \backslash\{0\}
$$

where $B$ is a subset of complex plane $\mathbb{C}$, called $q-$ geometric set if $q z \in B$, whenever $z \in B$. Note that if a subset $B$ of $\mathbb{C}$ is $q-$ geometric, then it contains all geometric sequences $\left\{z q^{n}\right\}_{0}^{\infty}, z q \in B$. Obviously, $D_{q} f(z) \rightarrow f^{\prime}(z)$ as $q \rightarrow 1^{-}$. The $q$ - difference operator (1.1) is sometimes called Jackson $q$ - difference operator. Note that such an operator plays an important role in the theory of hypergeometric series and quantum physics (see for instance $[1,4,5,10]$ ).

Also, note that $D_{q} f(0) \rightarrow f^{\prime}(0)$ as $q \rightarrow 1^{-}$and $D_{q}^{2} f(z)=D_{q}\left(D_{q} f(z)\right)$. In fact, $q-$ calculus is ordinary classical calculus without the notion of limits. Recent interest in $q-$ calculus is because of its applications in various branches of mathematics and physics. For definition and properties of $q$ - difference operator and $q-$ calculus, one may refer to $[1,4,5,10]$.

Under the hypothesis of the definition of $q$ - difference operator, then we have the following rules:

(1) For a function $f(z)=z^{n}$, we observe that

$$
D_{q} z^{n}=\frac{1-q^{n}}{1-q} z^{n-1}
$$

Therefore we have

$$
D_{q} f(z)=1+\sum_{n=2}^{\infty} a_{n} \frac{1-q^{n}}{1-q} z^{n-1} .
$$

(2) If functions $f$ and $g$ are defined on a $q-$ geometric set $B \subset \mathbb{C}$ such that $q-$ derivatives of $f$ and $g$ exist for all $z \in B$, then

(i) $D_{q}(a f(z) \pm b g(z))=a D_{q} f(z) \pm b D_{q} g(z)$ where $a$ and $b$ are real or complex constants. 
(ii) $D_{q}(f(z) \cdot g(z))=g(z) D_{q} f(z)+f(q z) D_{q} g(z)$.

(iii) $D_{q}\left(\frac{f(z)}{g(z)}\right)=\frac{g(q z) D_{q} f(z)-f(q z) D_{q} g(z)}{g(z) g(q z)}, \quad g(z) g(q z) \neq 0$.

(iv) As a right inverse, Jackson introduced $q$-integral

$$
\int_{0}^{z} f(t) d_{q} t=z(1-q) \sum_{n=0}^{\infty} q^{n} f\left(z q^{n}\right)
$$

provided that the series converges.

The following theorem is an analogue of the fundamental theorem of calculus.

A. Theorem. ([10]) Let $f$ be a $q-$ regular at zero, defined on $q-$ geometric set $B$ containing zero. Define

$$
F(z)=\int_{c}^{z} f(\zeta) d_{q} \zeta, \quad(\zeta \in B)
$$

where $c$ is a fixed point in $B$, then $F$ is $q$ - regular at zero. Furthermore $D_{q} F(z)$ exists for every $z \in B$ and

$$
D_{q} F(z)=f(z)
$$

for every $z \in B$.

Conversely, if $a$ and $b$ are two points in $B$, then

$$
\int_{a}^{b} D_{q} f(\zeta) d_{q} \zeta=f(b)-f(a) .
$$

(3) The $q-$ differential is defined as

$$
d_{q} f(z)=f(z)-f(q z) .
$$

Therefore we can write

$$
d_{q} f(z)=\frac{f(z)-f(q z)}{(1-q) z} d_{q} z .
$$

(4) The partial $q$ - derivative of a multivariable real continous functions $f\left(x_{1}, x_{2}, \ldots, x_{i-1}, x_{i}, x_{i+1}, \ldots, x_{n}\right)$ to a variable $x_{i}$ is defined by

$$
\begin{gathered}
D_{q, x_{i}} f(\vec{x})=\frac{f(\vec{x})-\varepsilon_{q, x_{i}} f(\vec{x})}{(1-q) x_{i}}, \quad x_{i} \neq 0, q \in(0,1) \\
{\left[D_{q, x_{i}} f(\vec{x})\right]_{x_{i}=0}=\lim _{x_{i} \rightarrow 0} D_{q, x_{i}} f(\vec{x})}
\end{gathered}
$$

where $\varepsilon_{q, x_{i}} f(\vec{x})=f\left(x_{1}, x_{2}, \ldots, x_{i-1}, q x_{i}, x_{i+1}, \ldots, x_{n}\right)$ and we use $D_{k, x}^{k}$ instead of operator $\frac{\partial_{q}^{k}}{\partial_{q} x^{k}}$ for some simplification.

Finally, let $\Omega$ be the family of functions $\phi$ analytic in $\mathbb{D}$, and satisfy the conditions $\phi(0)=0,|\phi(z)|<1$ for all $z \in \mathbb{D}$. Denote by $\mathcal{P}_{q}$ the family of functions $p$ of the form $p(z)=1+p_{1} z+p_{2} z^{2}+\cdots$, analytic in $\mathbb{D}$ and satisfy the condition

$$
\left|p(z)-\frac{1}{1-q}\right| \leq \frac{1}{1-q}, \quad z \in \mathbb{D}
$$

where $q \in(0,1)$ is a fixed real number. Let $\mathcal{A}$ be the family of functions $f$, defined by $f(z)=z+a_{2} z^{2}+a_{3} z^{3}+\ldots$, that are analytic in $\mathbb{D}$ and satisfy the conditions $f(0)=$ $0, f^{\prime}(0)=1$. If $f$ satisfies the condition

$$
1+\frac{1}{b}\left(q z \frac{D_{q}\left(D_{q} f(z)\right)}{D_{q} f(z)}\right) \prec \frac{1+z}{1-q z},
$$

where $b \in \mathbb{C}, b \neq 0$, then $f$ is called $q$ - convex function of complex order, and the class of such functions are denoted by $\mathcal{C}_{q}(b)$. If $f_{1}$ and $f_{2}$ are analytic functions in $\mathbb{D}$, then we say 
that $f_{1}$ is subordinate to $f_{2}$, written as $f_{1} \prec f_{2}$ if there exists a Schwarz function $\phi \in \Omega$ such that $f_{1}(z)=f_{2}(\phi(z)), z \in \mathbb{D}$. We also note that if $f_{2}$ univalent in $\mathbb{D}$, then $f_{1} \prec f_{2}$ if and only if $f_{1}(0)=f_{2}(0)$ and $f_{1}(\mathbb{D}) \subset f_{2}(\mathbb{D})$. This implies that $f_{1}\left(\mathbb{D}_{r}\right) \subset f_{2}\left(\mathbb{D}_{r}\right)$, where $\mathbb{D}_{r}=\{z:|z|<r, 0<r<1\}$ (Subordination principle [6] ).

We also need the following lemmas:

1.1. Lemma. Let $\phi$ be analytic in $\mathbb{D}$ with $\phi(0)=0$ and $|\phi(z)|<1, z \in \mathbb{D}$. If $|\phi(z)|$ attains its maximum value on the circle $|z|=r$ at a point $z_{0}$, then we have

$$
z_{0} \phi^{\prime}\left(z_{0}\right)=m \phi\left(z_{0}\right), \quad m \geq 1 .
$$

For more details of Jack's lemma, one may refer to [7].

1.2. Lemma. ([12]) If $h$ is an element of $\mathcal{C}_{q}(b)$, then

$$
F_{2}(|b|, R e b, q, r) \leq\left|D_{q} h(z)\right| \leq F_{1}(|b|, R e b, q, r)
$$

where

$$
\begin{aligned}
& F_{1}(|b|, R e b, q, r)=\left[(1-q r)^{R e b+|b|} \cdot(1+q r)^{R e b-|b|}\right]^{-\frac{1-q^{2}}{2 q^{2} \log q^{-1}}}, \\
& F_{2}(|b|, \operatorname{Re}, q, r)=\left[(1-q r)^{R e b-|b|} \cdot(1+q r)^{R e b+|b|}\right]^{-\frac{1-q^{2}}{2 q^{2} \log q^{-1}}} .
\end{aligned}
$$

The aim of this paper is to investigate properties of the class of $q-$ harmonic functions for which analytic part is $q$ - convex functions of complex order defined by

$\mathcal{S}_{\mathcal{H C}_{q}(b)}=\left\{f=h+\bar{g}: w_{q}(z)=\frac{D_{q} g(z)}{D_{q} h(z)}=\frac{\bar{f}_{\bar{z}}}{f_{z}}, w_{q}(z) \prec b_{1} \frac{1+z}{1-q z},\left|w_{q}(z)\right|<1, h \in \mathcal{C}_{q}(b)\right\}$,

where

$$
D_{q} h(z)=\frac{h(z)-h(q z)}{(1-q) z}=f_{z} \quad \text { and } \quad D_{q} g(z)=\frac{g(z)-g(q z)}{(1-q) z}=\bar{f}_{\bar{z}}
$$

\section{Main Results}

In this section, we first assume that the function $f$ is sense-preserving $q-$ harmonic function if and only if $w_{q}(z)=\frac{\bar{f}_{\bar{z}}}{f_{z}}$ is analytic. To show that

$(\Rightarrow)$ Let $f=h+\bar{g}$ be sense-preserving $q$ - harmonic function, then we will show that $w_{q}$ is analytic. Since $h(z)=z+\sum_{n=2}^{\infty} a_{n} z^{n}$ and $g(z)=\sum_{n=1}^{\infty} b_{n} z^{n}$ are analytic functions, then we can write $q$ - derivatives of these functions as

$$
D_{q} h(z)=1+\sum_{n=2}^{\infty} \frac{1-q^{n}}{1-q} a_{n} z^{n-1} \quad \text { and } \quad D_{q} g(z)=b_{1}+\sum_{n=2}^{\infty} \frac{1-q^{n}}{1-q} b_{n} z^{n-1} \text {. }
$$

We must note that when $q \rightarrow 1^{-}, D_{q} h(z)$ reduces to $h^{\prime}(z)$ and $D_{q} g(z)$ reduces to $g^{\prime}(z)$. The second $q-$ dilatation and $q-$ Jakobian are defined by

$$
\begin{gathered}
w_{q}(z)=\frac{D_{q} g(z)}{D_{q} h(z)}=\frac{\bar{f}_{\bar{z}}}{f_{z}}, \\
J_{f q}(z)=\left|D_{q} h(z)\right|^{2}-\left|D_{q} g(z)\right|^{2} .
\end{gathered}
$$

Also, the total $q$ - differential of $f(\vec{x})$ can be written in the following manner,

$$
d_{q} f(\vec{x})=D_{q, x_{1}} d_{q} x_{1}+D_{q, x_{2}} d_{q} x_{2}+D_{q, x_{3}} d_{q} x_{3}+\cdots+D_{q, x_{n}} d_{q} x_{n} .
$$

Therefore the $q$ - differential can be written as

$$
d_{q} f=D_{q, z} d_{q} z+D_{q, \bar{z}} d_{q} \bar{z} .
$$


Consequently, $f$ is locally univalent and sense-preserving if $\left|D_{q} h(z)\right|>\left|D_{q} g(z)\right|$ and sense-reversing if $\left|D_{q} g(z)\right|>\left|D_{q} h(z)\right|$. Note that $f_{z} \neq 0$ whenever $J_{f q}(z)>0$. For sense-preserving $f$, one sees that

$$
\left(\left|D_{q} h(z)\right|-\left|D_{q} g(z)\right|\right)\left|d_{q} z\right| \leq\left|d_{q} f\right| \leq\left(\left|D_{q} h(z)\right|+\left|D_{q} g(z)\right|\right)\left|d_{q} z\right| .
$$

With aid of these definitions, let $f=h+\bar{g}$ be the solution of the non-linear elliptic partial differential equation

$$
w_{q}(z) f_{z}=\bar{f}_{\bar{z}}
$$

under the condition $\left|w_{q}(z)\right|<1$ for all $z \in \mathbb{D}$. A non-constant complex -valued function $f$ is $q$ - harmonic and orientation sense-preserving mapping on $\mathbb{D}$ if and only if $f$ is the solution of the non-linear elliptic partial differential equation

$$
w_{q}(z) f_{z}=\bar{f}_{\bar{z}}
$$

where

$$
f_{z}=D_{q} h(z)=\frac{h(z)-h(q z)}{(1-q) z} \quad \text { and } \quad \bar{f}_{\bar{z}}=D_{q} g(z)=\frac{g(z)-g(q z)}{(1-q) z} .
$$

If we take the $q$ - derivative of equation $(2.1)$ with respect to $\bar{z}$, we get

$$
\bar{f}_{\bar{z} z}=f_{z \bar{z}} w_{q}(z)+f_{z} \frac{\partial w_{q}}{\partial \bar{z}} .
$$

On the other hand, since $f$ is $q-$ harmonic, then we have $\triangle f=4 \frac{\partial^{2} f}{\partial z \partial \bar{z}}=4 f_{z \bar{z}}=0$ and $\bar{f}_{\bar{z} z}=0$. Therefore the equality $(2.2)$ reduces to

$$
f_{z} \frac{\partial w_{q}}{\partial \bar{z}}=0
$$

and this shows that $\frac{\partial w_{q}}{\partial \bar{z}}=0$, that is, $w_{q}$ is analytic.

$(\Leftarrow)$ Conversely, if $w_{q}$ is analytic in $\mathbb{D}$, then $\frac{\partial w_{q}}{\partial \bar{z}}=0$. Therefore equality $(2.2)$ reduces to

$$
\bar{f}_{\bar{z} z}=f_{z \bar{z}} w_{q}(z)
$$

On the other hand, using the definition of $w_{q}$, we have $\left|w_{q}(z)\right|<1$. Thus, we get

$$
1-\left|w_{q}(z)\right| \neq 0 \text {. }
$$

Considering (2.4) and (2.5), we obtain

$$
\bar{f}_{\bar{z} z}=f_{z \bar{z}} w_{q}(z) \Rightarrow f_{z \bar{z}}=0
$$

and the equality (2.6) shows that $f$ is $q$ - harmonic. This proves our assumption.

We now investigate properties of the class $\mathcal{S}_{\mathcal{H}} e_{q}(b)$. For Theorem 2.4, we need the following results. The first theorem is very important in order to obtain subordination of the analytic functions involving $q$ - difference operator.

2.1. Theorem. ([2]) $p$ is an element of $\mathcal{P}_{q}$ if and only if $p(z) \prec \frac{1+z}{1-q z}$. This result is sharp for the functions $p(z)=\frac{1+\phi(z)}{1-q \phi(z)}$, where $\phi$ is a Schwarz function.

Proof. If $p$ is an element of $\mathcal{P}_{q}$, then we have

$$
\left|p(z)-\frac{1}{1-q}\right| \leq \frac{1}{1-q} \Leftrightarrow|p(z)-m| \leq m,
$$

where $m=\frac{1}{1-q}>1$. Therefore we can write

$$
\left|\frac{1}{m} p(z)-1\right| \leq 1
$$


Thus the function $\psi(z)=\frac{1}{m} p(z)-1$ is analytic and has modulus at most 1 in $\mathbb{D}$, and so

$$
\phi(z)=\frac{\psi(z)-\psi(0)}{1-\overline{\psi(0)} \psi(z)}=\frac{\left(\frac{1}{m} p(z)-1\right)-\left(\frac{1}{m}-1\right)}{1-\left(\frac{1}{m}-1\right)\left(\frac{1}{m} p(z)-1\right)}
$$

satisfies the conditions of Schwarz lemma. This shows that we can write

$$
p(z)=\frac{1+\phi(z)}{1-\left(1-\frac{1}{m}\right) \phi(z)} \Rightarrow p(z) \prec \frac{1+z}{1-q z} .
$$

Conversely, suppose that the function $p$ is analytic in $\mathbb{D}$ and satisfies the condition $p(0)=$ 1 and

$$
\begin{aligned}
p(z) \prec \frac{1+z}{1-q z} \Rightarrow p(z) & =\frac{1+\phi(z)}{1-\left(1-\frac{1}{m}\right) \phi(z)} \\
p(z)-m & =m \frac{\frac{1-m}{m}+\phi(z)}{1+\frac{1-m}{m} \phi(z)} .
\end{aligned}
$$

On the other hand the function $\frac{\frac{1-m}{m}+\phi(z)}{1+\frac{1-m}{m} \phi(z)}$ maps the unit circle onto itself, then we have

$$
|p(z)-m| \leq m \Leftrightarrow\left|p(z)-\frac{1}{1-q}\right| \leq \frac{1}{1-q} .
$$

This shows that $p \in \mathcal{P}_{q}$.

We must note that the linear tranformation $\frac{1+z}{1-q z}$ maps $|z|=r$ onto the disc with centre $C(r)=\frac{1+q r^{2}}{1-q^{2} r^{2}}$ and radius $\rho(r)=\frac{(1+q) r}{1-q^{2} r^{2}}$.

2.2. Lemma. If $f$ is a function (real or complex valued) defined on $q-$ geometric set $\mathbb{B}$ with $|q| \neq 1$, then

$$
D_{q}(\log f(z))=\frac{D_{q} f(z)}{f(z)} .
$$

Proof. Using the definition of $q$ - difference operator, then we have

$$
D_{q}(\log f(z))=\frac{\log f(q z)-\log f(z)}{q z-z}=\log \left(1+h \frac{D_{q} f(z)}{f(z)}\right)^{\frac{1}{h}} .
$$

If we take limit for $h \rightarrow 0$, we obtain the desired result.

2.3. Lemma. ( $q$-Jack's Lemma) Let $\phi$ be analytic in $\mathbb{D}$ with $\phi(0)=0$. If $|\phi(z)|$ attains its maximum value on the circle $|z|=r$ at a point $z_{0} \in \mathbb{D}$, then we have

$$
z_{0} D_{q} \phi\left(z_{0}\right)=m \phi\left(z_{0}\right)
$$

where $m \geq 1$ is a real number.

Proof. Using the definition of $q$ - difference operator and Jack's lemma, then we can write

$$
D_{q} \phi(z)=\frac{\phi(z)-\phi(q z)}{z-q z}=\frac{\phi(z)-\phi\left(z_{0}\right)}{z-z_{0}}, \quad q z=z_{0} .
$$

If we take limit for $z \rightarrow z_{0}$, we get

$$
\lim _{z \rightarrow z_{0}} D_{q} \phi(z)=D_{q} \phi\left(z_{0}\right)=\lim _{z \rightarrow z_{0}} \frac{\phi(z)-\phi\left(z_{0}\right)}{z-z_{0}}=\phi^{\prime}\left(z_{0}\right) .
$$

Therefore we have

$$
z_{0} D_{q} \phi\left(z_{0}\right)=m \phi\left(z_{0}\right)
$$


2.4. Theorem. If $f=h+\bar{g}$ is an element of $\mathcal{S}_{\mathcal{H}} e_{q}(b)$, then

$$
\frac{g(z)}{h(z)} \prec b_{1} \frac{1+z}{1-q z} \text {. }
$$

Proof. Since $f=h+\bar{g} \in \mathcal{S}_{\mathcal{H} e_{q}(b)}$, then we have

$$
\frac{D_{q} g(z)}{D_{q} h(z)} \prec b_{1} \frac{1+z}{1-q z} .
$$

The linear transformation $w=b_{1} \frac{1+z}{1-q z}$ maps $|z|=r$ onto the disc with centre $C(r)=$ $\left(\frac{\alpha_{1}\left(1+q r^{2}\right)}{1-q^{2} r^{2}}, \frac{\alpha_{2}\left(1+q r^{2}\right)}{1-q^{2} r^{2}}\right)$ and radius $\rho(r)=\frac{\left|b_{1}\right|(1+q) r}{1-q^{2} r^{2}}$, where $\alpha_{1}=R e b_{1}$ and $\alpha_{2}=R e b_{2}$. Thus using the subordination principle and the definition of the class $\mathcal{S}_{\mathcal{H}} e_{q}(b)$, we can write

$$
w_{q}\left(\mathbb{D}_{r}\right)=\left\{\frac{D_{q} g(z)}{D_{q} h(z)}:\left|\frac{D_{q} g(z)}{D_{q} h(z)}-\frac{b_{1}\left(1+q r^{2}\right)}{1-q^{2} r^{2}}\right| \leq \frac{\left|b_{1}\right|(1+q) r}{1-q^{2} r^{2}}, q \in(0,1)\right\} .
$$

In order to verify Schwarz function conditions, we define the function $\phi$ by

$$
\frac{g(z)}{h(z)}=b_{1} \frac{1+\phi(z)}{1-q \phi(z)} \text {. }
$$

Note that $\phi$ is a well defined analytic function and

$$
\left.\frac{g(z)}{h(z)}\right|_{z=0}=b_{1}=b_{1} \frac{1+\phi(0)}{1-q \phi(0)} \text {. }
$$

This proves that $\phi(0)=0$. We now need to show that $|\phi(z)|<1$ for all $z \in \mathbb{D}$. If we take $q-$ derivative of both sides of (2.9) and simplify, we get

$$
\frac{D_{q} g(z)}{h(z)}-\frac{g(q z) D_{q} h(z)}{h(z) h(q z)}=b_{1} \frac{D_{q} \phi(z)-q \phi(q z) D_{q} \phi(z)+q D_{q} \phi(z)+q \phi(q z) D_{q} \phi(z)}{(1-\phi(z))(1-\phi(q z))} .
$$

Multiplying both sides of this equation by $h(z) / D_{q} h(z)$ and simplifying, we obtain

$$
\frac{D_{q} g(z)}{D_{q} h(z)}=b_{1}\left(\frac{1+\phi(q z)}{1-q \phi(q z)}+\frac{(1+q) z D_{q} \phi(z)}{(1-q \phi(z))(1-q \phi(q z))} \cdot \frac{h(z)}{z D_{q} h(z)}\right) .
$$

Applying Lemma 2.2 in the equation (2.10), we can write the following form

$$
\frac{D_{q} g(z)}{D_{q} h(z)}=b_{1}\left(\frac{1+\phi(q z)}{1-q \phi(q z)}+\frac{(1+q) z D_{q} \phi(z)}{(1-q \phi(z))(1-q \phi(q z))}(1-q \phi(z))^{b \frac{1-q^{2}}{q^{2} \log q^{-1}}}\right) .
$$

Assume to the contrary that there esists a point $z_{0} \in \mathbb{D}_{r}$ such that $\left|\phi\left(z_{0}\right)\right|=1$. In view of Lemma 2.3, equation (2.11) gives

$\frac{D_{q} g\left(z_{0}\right)}{D_{q} h\left(z_{0}\right)}=b_{1}\left(\frac{1+\phi\left(q z_{0}\right)}{1-q \phi\left(q z_{0}\right)}+\frac{(1+q) m \phi\left(z_{0}\right)}{\left(1-q \phi\left(z_{0}\right)\right)\left(1-q \phi\left(q z_{0}\right)\right)}\left(1-q \phi\left(z_{0}\right)\right)^{b \frac{1-q^{2}}{q^{2} \log q^{-1}}}\right) \notin w_{q}\left(\mathbb{D}_{r}\right)$.

This contradicts our assumption (2.8) and therefore $|\phi(z)|<1$ for all $z \in \mathbb{D}$. This completes the proof of our theorem.

2.5. Corollary. If $f=h+\bar{g} \in \mathcal{S}_{\mathcal{H e}_{q}(b)}$, then we have

$$
F_{2}\left(|b|, \operatorname{Reb},\left|b_{1}\right|, q, r\right) \leq\left|D_{q} g(z)\right| \leq F_{1}\left(|b|, \operatorname{Reb},\left|b_{1}\right|, q, r\right),
$$

where

$$
\begin{aligned}
& F_{1}\left(|b|, R e b,\left|b_{1}\right|, q, r\right)=\left[(1-q r)^{R e b+|b|}(1+q r)^{R e b-|b|}\right]^{-\frac{1-q^{2}}{2 q^{2} \log q^{-1}}} \frac{\left|b_{1}\right|(1+r)}{1-q r}, \\
& F_{2}\left(|b|, R e b,\left|b_{1}\right|, q, r\right)=\left[(1-q r)^{R e b-|b|}(1+q r)^{R e b+|b|}\right]^{-\frac{1-q^{2}}{2 q^{2} \log q^{-1}}} \frac{\left|b_{1}\right|(1-r)}{1+q r} .
\end{aligned}
$$


Proof. Since $f=h+\bar{g}$ is an element of $\mathcal{S}_{\mathcal{H C}_{q}(b)}$, from Theorem 2.4 we write $\frac{D_{q} g(z)}{D_{q} h(z)} \prec$ $b_{1} \frac{1+z}{1-q z}$, where $h \in \mathcal{C}_{q}(b)$. Therefore we have

$$
\left|\frac{D_{q} g(z)}{D_{q} h(z)}-\frac{b_{1}\left(1+q r^{2}\right)}{1-q^{2} r^{2}}\right| \leq \frac{\left|b_{1}\right|(1+q) r}{1-q^{2} r^{2}} .
$$

This inequality yields

$$
\left|D_{q} g(z)\right| \leq\left|D_{q} h(z)\right| \frac{\left|b_{1}\right|(1+r)}{1-q r} .
$$

If we use Lemma 1.2, we get the right side of (2.12). Similarly, we can prove the other side of the inequality (2.12).

2.6. Corollary. If $f=h+\bar{g} \in \mathcal{S}_{\mathcal{H} e_{q}(b)}$, then we have

$$
f=h(z)+\overline{h(z) b_{1} \frac{1+\phi(z)}{1-q \phi(z)}}
$$

where $\phi$ is a Schwarz function.

Proof. Using Theorem 2.4, then we can write

$$
\frac{g(z)}{h(z)} \prec b_{1} \frac{1+z}{1-q z} \Rightarrow \frac{g(z)}{h(z)}=b_{1} \frac{1+\phi(z)}{1-q \phi(z)} .
$$

Therefore we obtain

$$
g(z)=h(z) b_{1} \frac{1+\phi(z)}{1-q \phi(z)}
$$

which gives (2.13).

\section{References}

[1] Andrews, G.E. Applications of basic hypergeometric functions, SIAM Rev. 16 (1974), 441484.

[2] Çetinkaya, A. and Mert, O. A certain class of harmonic mappings related to functions of bounded boundary rotation, Proc. of $12^{\text {th }}$ Symposium on Geometric Function Theory and Applications (2016), 67-76.

[3] Duren, P. Harmonic mappings in the plane, Cambridge Tracts in Math. 2004.

[4] Fine, N.J. Basic hypergeometric series and applications, Math. Surveys Monogr. 1988.

[5] Gasper, G. and Rahman, M. Basic hypergeometric series, Cambridge University Press, 2004.

[6] Goodman, A.W. Univalent functions Volume I and II, Polygonal Pub. House, 1983.

[7] Jack, I.S. Functions starlike and convex of order $\alpha$, J. Lond. Math. Soc. (2), 3 (1971), 469-474.

[8] Jackson, F.H. On $q-$ functions and a certain difference operator, Trans. Roy. Soc. Edin. 46 (1908), 253-281.

[9] Jackson, F.H. On q-difference integrals, Quart. J. Pure Appl. Math. 41 (1910), 193-203.

[10] Kac, V. and Cheung, P. Quantum calculus, Springer, 2001.

[11] Lewy, H. On the non-vanishing of the Jacobian in certain one-to-one mappings, Bull. Amer. Math. Soc. 42 (1936), 689-692.

[12] Polatog̃lu, Y., Aydog̃an, M. and Mert, O. Some properties of $q-$ convex functions of complex order, Sarajevo J. Math. Submitted, 2016. 